\title{
Essais
}

Revue interdisciplinaire d'Humanités

Narration et lien social

\section{Susan Hapgood, Cornelia Lauf, In deep: certificates of authenticity in art}

François Trahais

\section{OpenEdition}

Édition électronique

URL : https://journals.openedition.org/essais/9613

DOI : 10.4000/essais. 9613

ISSN : 2276-0970

Éditeur

École doctorale Montaigne Humanités

\section{Édition imprimée}

Date de publication : 15 mai 2013

Pagination : 221-224

ISBN : 978-2-9544269-0-7

ISSN : 2417-4211

\section{Référence électronique}

François Trahais, "Susan Hapgood, Cornelia Lauf, In deep: certificates of authenticity in art », Essais [En ligne], 3 | 2013, mis en ligne le 01 septembre 2021, consulté le 08 septembre 2021. URL : http:// journals.openedition.org/essais/9613; DOI : https://doi.org/10.4000/essais.9613 
tant que stratégie intellectuelle ${ }^{8}$. Est-il également besoin de souligner l'apport de l'histoire transnationale dans ce processus d'appropriation car il y a des phénomènes historiques qui la notion même de frontière, qui apparait elle même comme un objet à déconstruire. On reconnaît nettement l'ambition affichée dans cet ouvrage qui est de faire l'histoire de ce concept. Cependant, si cela n'est pas explicitement formulé par l'auteur, force est de constater qu'il s'agit d'un aveu à demi mot. Sans doute cela s'explique t-il par une prudence qui voudrait que le sociologue n'empiète pas sur les plates bandes de l'historien. C'est pourtant Garcia Bilbao lui-même qui, dans ses pages rappelle, la nécessité d'approcher le concept de migration « contraintes » sous l'angle de la transdisciplinarité ! (Garcia Bilbao, ch. 6)

Aubin Gonzalez

EA $4574 \mathrm{SPH}$

Université Michel de Montaigne Bordeaux 3

aubin.gonzalez@etu.u-bordeaux3.fr

\begin{abstract}
François Trahais, Catalogue d'exposition réalisé par Susan Hapgood, Cornelia Lauf (éd.), In deep: certificates of authenticity in art, Roma publications, Amsterdam et SBKM / De Vleeshal, Middelburg, 2011. Langue : anglais, $102 \mathrm{p}$.
\end{abstract}

Pour la première fois dans l'histoire des catalogues d'exposition, un recensement exhaustif des certificats d'authenticité dans l'art du $\mathrm{XX}^{\mathrm{e}}$ siècle vient d'être édité. Témoignant d'une exposition ayant récemment circulé à travers l'Europe, les États-Unis et l'Asie, l'ouvrage se caractérise par la richesse de ses illustrations en couleur. De manière inédite, un corpus de cinquantedeux contrats de cession, protocoles de réalisation et de certificats d'authenticité y est présenté. Ces " énoncés d'art " tel que les nomment de manière générique Ghislain Mollet-Viéville et Jean-Baptiste Farkas', s’inscrivent dans l'histoire de la dématérialisation de l'œuvre. Avec l'invention du readymade par Marcel Duchamp au début du siècle dernier et le développement d'une certaine forme de conceptualisme, l'idée prime désormais sur l'objet dans l'histoire de la modernité. D’autant plus que les nouvelles techniques de reproductibilité de l'œuvre contribuent elles aussi à discuter les traditionnels critères d'authenticité. Les conditions de matérialité et d'originalité sont alors remises en question. Le statut de l'auteur quant à lui se repense en fonction de la participation et de

8 Selon le titre de l'ouvrage de Nancy Green, Repenser les Migrations, PUF, Paris, 2002.

9 G. Mollet-Viéville \& J.-B. Farkas, "À propos des "énoncés d'art" ", in Critique, Les Édition de Minuit, août-septembre 2010, p. 719-734. 
l'implication du spectateur dans le processus de création. Aussi les contrats, les protocoles et les certificats accompagnent désormais, de façon presque systématique, les œuvres d'art contemporain. Il était donc urgent pour l'histoire de l'art de disposer d'un premier inventaire de ces nouveaux types de documents.

L'iconographie du catalogue bénéficie de techniques d'impressions d'une grande qualité. Photographiés en couleur, les contrats et les certificats y sont traités tels des reproductions d'œuvre d'art. Les reproductions sont nettes et détaillées. Les légendes n'oublient aucun élément de référence. De manière encore plus spécifique, le format du catalogue $(26,7$ x 18,7 cm) permet d'envisager ces documents dans leurs dimensions réelles. Les certificats de Dan Flavin ou les diagrammes de Sol LeWitt s'accompagnent de croquis colorés, ce qui nous éclaire sur leurs manières de travailler ainsi que sur la rigueur des projets. Les reçus d'une "Zone de sensibilité Picturale Immatérielle » élaborés par Yves Klein dès 1959, se composent, pour moitié, d'un aplat en IKB (International Klein Blue) et le soin tout particulier accordé à l'allure du document prolonge et répond à la démarche dont il est question. Qu'ils soient jaunis, qu'ils soient tachés ou volontairement colorés, ces contrats se déclinent tout en restant fidèles aux principes du genre. En effet, la trame du certificat garantie l'unité et la logique d'un corpus visuel très hétérogène. Mais l'on peut cependant regretter qu'un ouvrage retraçant l'histoire de ce type d'écrit ait été ordonné par ordre alphabétique. Un classement chronologique aurait mieux convenu pour répondre au projet de l'exposition, notamment parce qu'il pourrait permette au lecteur d'observer une esthétisation progressive de cette documentation. La richesse du chromatisme, l'ornementation même très épurée ainsi que les procédés de mise en page reproduisent une certaine forme de fétichisation : celle de la page écrite, imprimée ou manuscrite. Ce livre présente donc plus des images que des archives. Alors que la dématérialisation de l'art visait à libérer l'œuvre des principes matériels et décoratifs, de nouveaux certificats reprennent à leur compte ces propriétés. Le paradoxe est encore plus flagrant lorsque l'on sait que c'est en voulant échapper au marché de l'art que le conceptualisme s'est doté d'un outil comparable au contrat de vente.

Par ailleurs, les quatre textes du catalogue explorent de façon singulière et de manière complémentaire l'histoire des énoncés d'art. En lien direct avec l'histoire du droit d'auteur, les commentaires critiques de ces documents sont formulés selon quatre points de vue. Dans son article intitulé "White is the color: the conceptualization of the art space ", Lorenzo Benedetti, actuel directeur du centre d'art de Vleeshal, à Middelburg, aux Pays-Bas, souligne l'importance de ces énoncés dans le champ muséal. Le certificat se présente, selon lui, comme un moyen de "conceptualiser l'espace d'exposition". Il anticipe les questions techniques et programme les modalités d'accrochage et de présentation des œuvres. Par ailleurs, l'article de Susan Hapgood et de Cornelia Lauf intitulé "Doodle, deeds, and claims» retrace et analyse les différents processus de déma- 
térialisation de l'œuvre, depuis Marcel Duchamp jusqu’à Simon Starling, en passant par Yves Klein. Selon ces deux commissaires d'exposition, le recours aux contrats ou aux brevets s'est imposé dans la cadre d'une démystification progressive du statut de l'auteur. Le troisième article intitulé "Authenticity in art and law: a question of attribution or autorisation ? " fut signé par Daniel McClean. Ce commissaire d'exposition indépendant y rappelle les traditionnels principes justifiant la supériorité de l'original sur la copie. Depuis la Renaissance et avec la Révolution Industrielle, des procédés techniques de reproductibilité de l'œuvre sont inventés. En se référant aux écrits de Walter Benjamin ${ }^{10}$, Daniel McClean rappelle que la fabrication d'œuvre en série par le biais de la gravure, de la lithographie, de la sérigraphie, de la photographie a contribué à affaiblir la suprématie de l'œuvre unique. Mais le critère d'originalité fut cependant protégé par la numérotation presque systématique de ces réalisations. Participant à cultiver la rareté en tant que qualité, les principes spéculatifs se sont développés au cours de la période dite moderne. Daniel McClean souligne enfin l'importance du Minimalisme dans le processus de démystification de l'auteur. Les objets spécifiques de l'art minimal ne nécessitent pas d'être matériellement produits par les artistes. En effet, les techniques venant de l'industrie répondent davantage aux exigences formelles de l'art minimal. Donald Judd, Dan Flavin ou encore Sol LeWitt sollicitèrent dès lors des professionnels de la technique, afin de réaliser leurs projets. C'est pourquoi survint la nécessité d'encadrer d'un point de vue contractuel la collaboration de l'auteur et de son exécutant. Le droit de la propriété intellectuelle définit, dès lors, la distinction entre l'auteur, le co-auteur et l'interprète dans le champ des arts plastiques. Cependant, les nombreux litiges qui ont parfois opposé les artistes à leurs acheteurs sont rappelés. McClean évoque à ce sujet les relations parfois conflictuelles qu'entretenait Giuseppe Panza avec Carl Andre ou Donald Judd. Sans autorisation préalable, le célèbre collectionneur lombard se permettait de produire des œuvres à partir des plans et des schémas qu'il avait acquis auprès de ces deux artistes. Enfin, en cette fin de XX siècle, le développement croissant de la contractualisation des œuvres en est relaté de manière plus énumérative dans le dernier texte du catalogue par Martha Buskirk, Professeure d'histoire de l'art au Montserrat College de Beverly, Massachusetts. À travers un essai intitulé "Certifiable ", Martha Buskirk clôture le catalogue en soulignant l'importance historique de deux types de contrat. Ce sont les "Avertissements " de Daniel Buren, que l'artiste conçoit avec l'aide de Michel Claura en 1968-1969, ainsi que le "Contrat pour la préservation des droits de l'artiste sur toute œuvre cédée " rédigé par Seth Siegelaub et Robert Projansky en 1971 qui inspirent les artistes et les marchands des deux côtés de l'Atlantique depuis plus de quarante ans. Les

10 W. Benjamin, L'ouvre d'art à l'époque de sa reproductibilité technique, nouvelle traduction de Lionel Duvoy de la $4^{\mathrm{e}}$ version de l'essai (1939) ainsi que les passages non conservés par Benjamin figurant dans la $2^{\mathrm{e}}$ version de l'essai (fin 1935-février 1936), Paris, Allia, 2012. 
modalités d'achat, d'exposition et de conservation se voient réglées avec rigueur et précision en intégrant la notion de droit moral dont la France fait partie des pays bénéficiaires. Et force est de constater, selon Martha Burskirk, l'incontournable et l'indispensable recours à ce nouveau type de certificat.

La dématérialisation de l'ouvre annoncée par Lucy Lippard et John Chandler dès $1968^{11}$ demeure une des problématiques centrales de la création actuelle et nous savons comment la disparition progressive du critère de matérialité profite au développement des énoncés d'art. Le catalogue In deed, Certificates of authenticity in art s'impose comme l'une des meilleures ressources iconographiques et protocolaires en matière de certificats d'authenticités. Aussi l'ouvrage peut-il être considéré comme une référence précieuse tant pour l'histoire de l'art que pour l'histoire du droit de la propriété intellectuelle.

François Trahais

EA 538 Histoire de l'art

Université Michel de Montaigne Bordeaux 3

f.trahais@gmail.com

\section{TITRES DES ARTICLES DU CATALOGUE}

Benedetti L., "White is the color: the conceptualization of the art space", in In deed: certificates of authenticity in art, Roma publications, Amsterdam, in co-production with SBKM / De Vleeshal, Middelburg, 2011, p. 75-76.

Hapgood S. \& Lauf C., "Doodle, deeds, and claims", in In deed: certificates of authenticity in art, Roma publications, Amsterdam, in co-production with SBKM / De Vleeshal, Middelburg, 2011, p. 77-86.

McClean D., "Authenticity in Art and Law: a question of attribution or authorization?", in In deed: certificates of authenticity in art, Roma publications, Amsterdam, in co-production with SBKM / De Vleeshal, Middelburg, 2011, p. 87-96.

Buskirk M., "Certifiable", in In deed: certificates of authenticity in art, Roma publications, Amsterdam, in co-production with SBKM / De Vleeshal, Middelburg, 2011, p. 97-102.

\section{LISTE DES EXPOSITIONS}

September 10-October 9, 2011. De Kabinetten van de Vleeshal, Middelburg, The Netherlands. October 14-November 4, 2011. Fondazione Bevilacqua La Masa, Venice.

November 18-December 16, 2011. Khoj International Artist's Association, New Delhi. January 13-February 10, 2012. Mumbai Art Room, Mumbai.

February 3-March 2, 2012. Nero Hq, Rome.

March 30-April 28, 2012. John M. Flaxman Library Special Collections, The School of the Art Institute of Chicago, Chicago.

Spring 2012. Salt Beyoğlu, Istanbul.

Fall 2012. The Drawing Center, New York.

11 L. Lippard \& J. Chandler, "The dematerialization of Art ", in Art International, février 1968. 